\title{
Study on wet sensitive characteristics of Colpitts oscillation circuit
}

\author{
Shuo Wang ${ }^{1,}$, Xiaofeng Meng ${ }^{1, b}$, Guohua Wang ${ }^{1}$, Rui Zheng ${ }^{1}$ and Yang Lv ${ }^{2}$ \\ ${ }^{1}$ Science and Technology on Inertial Laboratory, Beihang University, Beijng 100191, China \\ 2 96831Army, Beijing 100015, China \\ awangshuo.buaa@gmail.com, bmengxf@buaa.edu.cn
}

Keywords: dew point measurement, Colpitts circuit, quartz crystal

\begin{abstract}
Based on the phenomenon that Colpitts circuit cannot drive quartz crystal in liquid phase environment, this paper studies the wet sensitive characteristics of Colpitts oscillation circuit. And we designed a device used as humidity sensor. Peltier element was used to cool quartz crystal until dew occurred and quartz crystal worked in the liquid environment. Colpitts circuit stopped oscillation when dew occurred. At the same time the temperature of crystal surface was measured and the temperature was approximately regarded as the dew point. Through the theoretical analysis of Colpitts circuit characteristic and the humidity sensing properties of quartz crystal admittance, then combined with experimental data comparison, the wet sensitive characteristics of Colpitts circuit was proved to be feasible and accurate for dew point recognition from the qualitative point. The result is of practical importance for further quantitative analysis and developing sensitive circuit type dew point instrument.
\end{abstract}

\section{Introduction}

Humidity is a term for the amount of water vapour in the air and can be described in different ways such as absolute humidity, relative humidity, dew point temperature, mixing ratio and wet-bulb temperature ${ }^{[1]}$. Although humidity measurement seems simple, it involves very complex physical-chemical theoretical analysis and calculation. Thus it is always a worldwide problem in the measurement field, which undoubtedly puts forward a high requirement to humidity sensors ${ }^{[2]}$.

Humidity sensing mechanism and material are always the research priorities of humidity sensors. Dew-point hygrometer is regarded as the most precise humidity sensor nowadays ${ }^{[3]}$. However, it is difficult to reach dynamic balance state in a very low humidity environment. Because the thickness of dew layer will become thin with low water content, and water molecules will move slow as the temperature decreases continually. The improvement of humidity sensing characteristic and stability in wide humidity and temperature ranges is the main trend for humidity sensors research nowadays.

Since Colpitts circuit cannot drive quartz crystal in liquid phase environment, this paper studies the wet sensitive characteristics of Colpitts oscillation circuit. Combining the theoretical analysis and experimental results, it is verified that quartz crystal as a humidity sensing element in Colpitts circuit has great reliability and accuracy for dew point measurement.

\section{Experimental System}

The sensing device for dew point measurement is shown in Fig. 1, which consists of an AT-cut quartz crystal resonator with its resonant frequency of $6 \mathrm{MHz}$, a Peltier element and a heat-pipe radiator. AT-cut quartz crystal is stuck directly on the cold surface of Peltier element by a PTFE gasket. And the heat surface of Peltier element should be laid closely to the radiator so as to play a better cooling effect. Lead out two wires respectively from the two electrodes of quartz crystal for connecting to Colpitts circuit. At the same time we need two platinum thermal resistances as temperature sensors, one is affixed to the cold surface of Peltier element for acquiring the quartz crystal surface temperature according to the temperature difference between quartz crystal surface and Peltier element, the other is exposed to the experimental environment for providing the ambient temperature at the same time. The Peltier element is of TEC1-3104 type with the maximum power of 8.2W. PT100 platinum thermal resistance is used as the temperature sensor. The data acquisition card is 
PCI4712AS2 high speed data acquisition card. And VM2710 digital multimeter based on VXI bus is used to converse the resistance values of PT100 to temperature values with the measurement accuracy of $10^{-3}$.

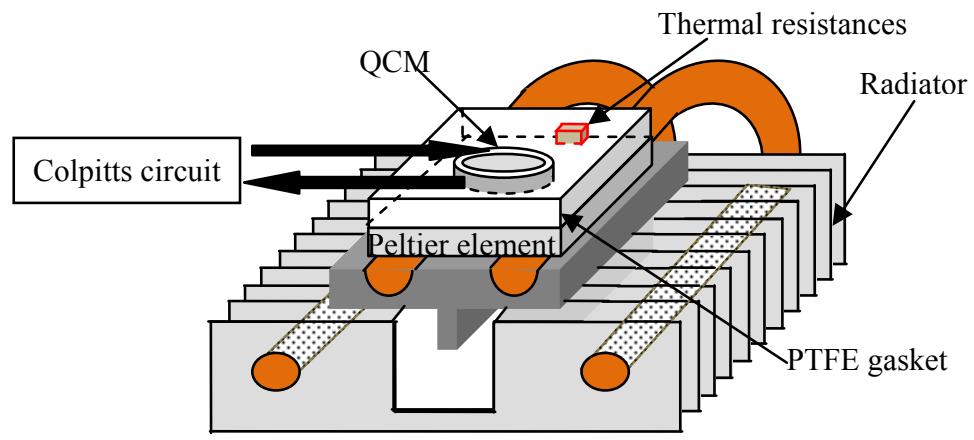

Fig. 1 Schematic diagram of the measurement system

\section{Measuring Principle}

Colpitts oscillation circuit analysis. Colpitts oscillation circuit is a positive feedback oscillation circuit based on the transistor. Thus, the circuit should simultaneously fulfill the amplitude condition and the phase condition to keep self-sustained oscillating. The basic expressions are as follows:

(1) Amplitude condition: the total loop gain $\mathrm{A}_{\mathrm{v}}{ }^{*} \mathrm{~A}_{\mathrm{f}}=1$,

(2) Phase condition: the total phase is $\mathrm{n}^{*} 360^{\circ} \quad(\mathrm{n}=0,1,2 \ldots)$.

$A_{v}$ is the amplification factor of basic amplifier circuit, and $A_{f}$ is the amplification factor of feedback loop. Fig. 2 shows the selected circuit, and the components parameters should be adjusted according to the resonance frequency of quartz crystal.

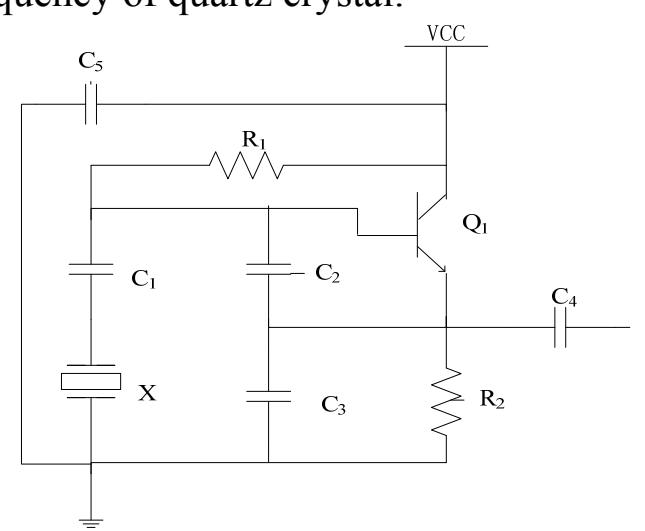

Fig. 2 Colpitts circuit diagram

Colpitts circuit has the advantage of high frequency stability. Therefore, it is usually utilized as the drive circuit for quartz crystal microbalance (QCM) ${ }^{[4,5]}$. However, Colpitts circuit will stop oscillating in the liquid phase environment. Because the characteristics of quartz crystal will change in the liquid, which makes the circuit not fulfill the oscillation conditions any more. And the phenomenon of circuit stopping oscillating is very sensitive. This paper utilizes this wet sensitive characteristic of Colpitts oscillation circuit to recognize the dew point.

Humidity sensing characteristic of quartz crystal. Dynamic inductance $\mathrm{L}_{\mathrm{q}}$ is large, typically from dozens of millihenries to dozens of henries. Dynamic capacitance $\mathrm{C}_{\mathrm{q}}$ is very small, typically in the magnitude of $10^{-3} \mathrm{PF}$. And dynamic resistance $\mathrm{R}_{\mathrm{q}}$ is small, usually from a few ohms to hundreds of ohms. Static capacitance $\mathrm{C}_{\mathrm{o}}$ is very small, generally about $2 \sim 5 \mathrm{PF}$. The quality factor $\mathrm{Q}_{\mathrm{q}}$ is as high as the magnitude of $10^{5} \sim 10^{6}$.

In order to get the reason why Colpitts oscillation circuit is hard to work in the liquid phase environment, we should firstly analyze the curve of quartz crystal admittance, as shown in Fig. 3. The equivalent resistance of quartz crystal will become large in the liquid. However, the gain of amplifier is limited. Thus, the amplitude condition cannot be satisfied any more. Furthermore, as the equivalent resistance and the static capacity increase in the liquid environment, the phase condition is also not 
satisfied. On the one hand, we can see that the radius of admittance circle will become smaller as the equivalent resistance increases. And the admittance circle will disjoint the horizontal axis when the equivalent resistance increases to a certain degree. Then the total phase of circuit will not satisfy the phase condition. On the other hand, the distance $\omega \mathrm{C}_{0}$ between the circle center and the horizontal will become large when the static capacity $\mathrm{C}_{0}$ increases. For this reason, the admittance circle will also gradually disjoint the horizontal axis. Therefore, the phase condition is also not satisfied when dew occurs on the quartz crystal surface. In conclusion, because of the amplitude characteristic and the phase characteristic, Colpitts circuit cannot work in the liquid environment, especially in the high viscosity liquid.

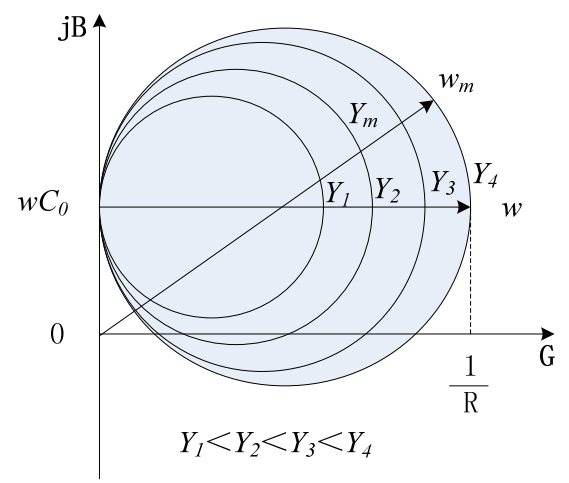

Fig. 3 Quartz crystal admittance curve

According to the equivalent circuit model of quartz crystal, the real part and the imaginary part of quartz admittance $Y=G+j B$ are:

$$
Y=R_{q} /\left[R_{q}^{2}+\left(L_{q} w-\frac{1}{C_{q} w}\right)^{2}\right]+j\left[\left(\frac{1}{C_{q} \omega}-L_{q} \omega\right) /\left[R_{q}^{2}+\left(L_{q} \omega-\frac{1}{C_{q} \omega}\right)^{2}\right]+\omega C_{0}\right] .
$$

From Eq. 1 we know that $G$ will reach the maximum when $L_{q} \omega-1 / C_{q} \omega=0$. The corresponding frequency of the maximum $G$ is the series resonance frequency, and the reciprocal of the maximum $G$ is the equivalent resistance $R_{q}$ under the series resonance frequency. The corresponding $B$ of the maximum $G$ is $\omega C_{0}$, thus the static capacitance $C_{0}$ can be acquired. During the process of humidity measurement in this paper, the quartz crystal surface experiences the dynamic process from dry to moisture condensation. Mechanical friction will cause the damping loss of crystal surface and the change of $R_{q}$ at the last period of this dynamic process. As a consequence, the amplitude characteristic of circuit will change and then lead to circuit stopping oscillating. As this paper aims to verify the reliability of gas-liquid phase change at the crystal surface when the circuit shops oscillating, the research is focused on the change trend of $R_{q}$ at the moment of circuit shops oscillating.

Fig. 4 (a) shows the varying curve of the real part of quartz admittance measured by the impedance analyzer under different conditions. It reveals that the conductance $G$ of quartz crystal has a shape decrease at the moment of circuit shopping oscillation. When cooling further continues, the condensation degree will continually deepen, and the equivalent resistance $R_{q}$ will increase, thus the conductance $G$ will further decrease. In order to analyze the varying trend of $R_{q}$ at the moment of circuit shopping oscillation and the sensitivity of dew recognition, $R_{q}$ of continuous 20 seconds is recorded during the former mentioned dynamic process. And the differences between the adjacent two values are calculated, which are shown in Fig. 4 (b). During this dynamic process, it experience 14 seconds from the start of cooling to the shop of circuit oscillation. The experimental data reveals that the difference of adjacent equivalent resistances reaches the maximum value at the fourteenth second. The analysis of experimental data corresponds with the experimental phenomenon. It is preliminarily demonstrated that the moment of circuit shopping oscillation is exactly the moment of gas-liquid phase transformation on the quartz surface. In order to further verify the reliability of Colpitts circuit used to recognize dew, we conduct five measurement processes under different humidity environment, and record $R_{q}$ under different stages. 


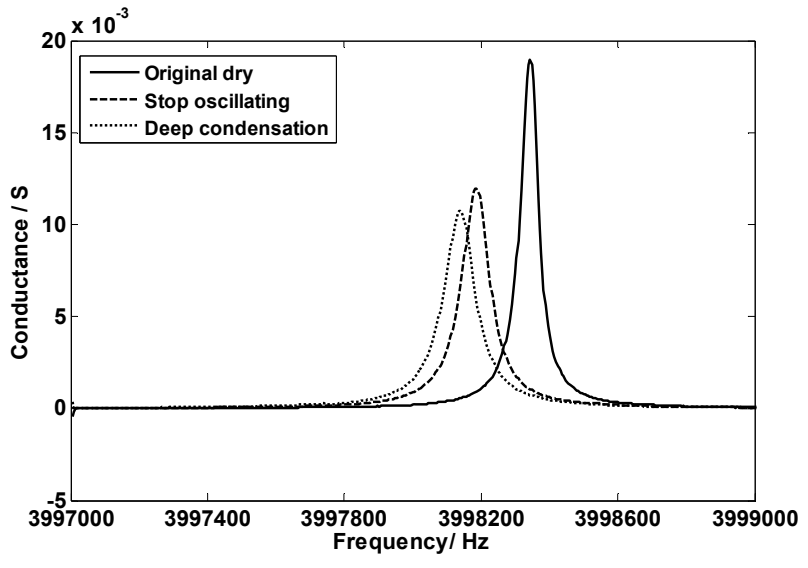

Fig. 4 (a) Conductance curve under different stages

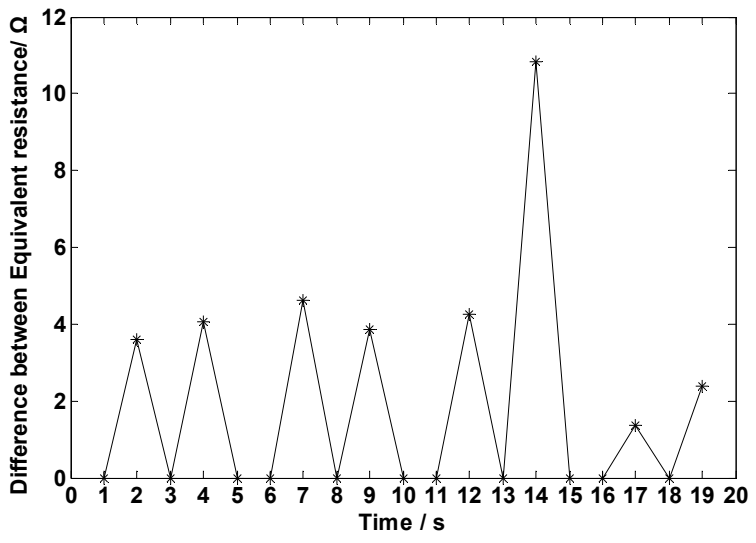

Fig. 4 (b) Equivalent resistance differences

Five groups of $R_{q}$ varying with the state of quartz surface are shown in Fig. 5. It demonstrates that the equivalent resistances at the moment of circuit shopping oscillation are of the same magnitude. Furthermore, the equivalent resistances under the original dry environment are also of the same magnitude. This experimental result can qualitatively prove the feasibility of the proposed method for dew point recognition.

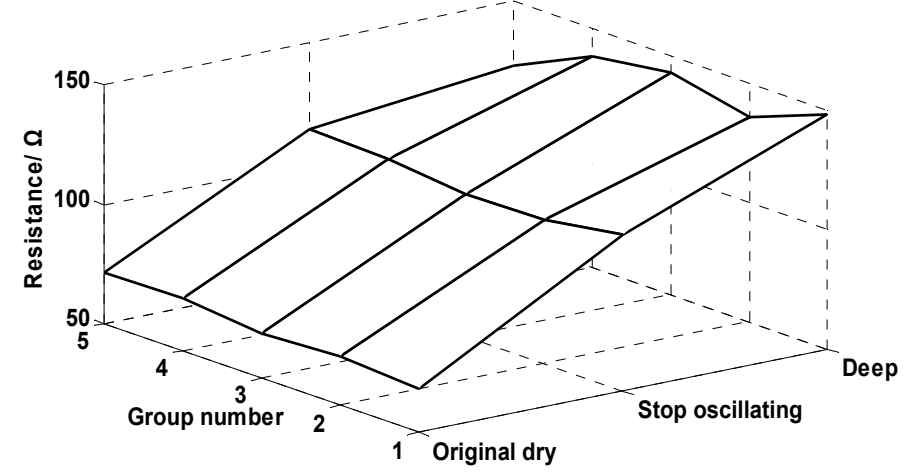

Fig. 5 Trend of quartz crystal equivalent resistance

\section{Measuring experiment}

Quartz crystal temperature measurement. The temperature measured by platinum thermal resistors is the temperature of Peltier element. In order to acquire the temperature of quartz crystal, the relationship between Peltier element and quartz crystal must be analyzed through experiment. And the experiment is conducted with a steady cooling power and under a steady ambient temperature. Through the data fitting of experiment results, the fitted curve of the relationship between quartz crystal surface temperature and Peltier element temperature is drawn in Fig. 6. And the cubic curve-fitting equation is $\mathrm{T}_{\text {crystal }}=0.0007591 \mathrm{~T}_{\text {Peltier }}{ }^{3}-0.05966 \mathrm{~T}_{\text {Peltier }}{ }^{2}+1.935 \mathrm{~T}_{\text {Peltier }}+3.587$. Quartz crystal surface temperature can be calculated based on the measured temperature value of Peltier element according to this equation.

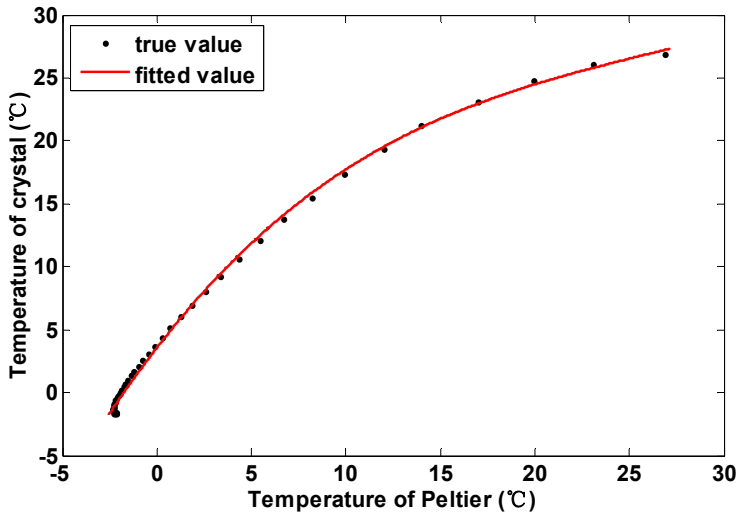


Fig. 6 Fitted curve of temperature

Humidity measurement. The measurement device is tested under seven different humidity environment supplied by the experimental box. The experimental box can offer steady ambient temperature and uniform humidity. The work current of Peltier element is $2 \mathrm{~A}$, and ambient temperature is $26.88^{\circ} \mathrm{C}$. The reference of environment humidity is supplied by capacitance moisture sensor HM1500 with the accuracy of $\pm 3 \% \mathrm{RH}$.

The measured data and the reference value under the seven different humidity environment are shown in Table 1. The result indicates that the error between the measured dew point temperature and the reference value is within $\pm 2.2^{\circ} \mathrm{C}$, and the error between the calculated measured relative humidity and the reference value is within $\pm 5.08 \%$. The comparison of the experiment results can qualitatively verify that the proposed method has certain accuracy for dew point measurement.

Table 1. Experimental results

\begin{tabular}{|c|c|c|c|c|c|c|}
\hline \multirow{2}{*}{ Number } & \multicolumn{3}{|c|}{ Temperature[ $\left.{ }^{\circ} \mathrm{C}\right]$} & \multicolumn{3}{c|}{ Relative humidity[\%] } \\
\cline { 2 - 7 } & $\mathrm{T}_{\text {reference }}$ & $\mathrm{T}_{\text {crystal }}$ & Error & $\mathrm{RH}_{\text {reference }}$ & $\mathrm{RH}_{\text {measured }}$ & Error \\
\hline 1 & 10.54 & 8.34 & 2.2 & 35.93 & 31 & 4.93 \\
\hline 2 & 14.13 & 12.35 & 1.78 & 45.52 & 40.52 & 5.0 \\
\hline 3 & 18.7 & 17.57 & 1.13 & 60.89 & 56.72 & 4.17 \\
\hline 4 & 20.03 & 19.64 & 0.39 & 66.13 & 64.56 & 1.57 \\
\hline 5 & 22.54 & 21.43 & 1.11 & 77.18 & 72.1 & 5.08 \\
\hline 6 & 23.12 & 22.14 & 0.98 & 79.92 & 75.31 & 4.61 \\
\hline 7 & 23.95 & 24.28 & -0.33 & 84.0 & 85.7 & -1.7 \\
\hline
\end{tabular}

\section{Conclusions}

This paper theoretically analyzes the Colpitts circuit condition of self-sustaining oscillation and the humidity sensing properties of quartz crystal admittance, which verifies the feasibility of quartz crystal as a humidity sensing element in Colpitts circuit. Experimentally, the reliability and accuracy of the proposed method are proved by comparison between the measured and reference data. This thesis possesses important theory significance on the study of new dew point hygrometer.

\section{Acknowledgments}

This paper is supported by the National Natural Science Foundation Innovation Group of China (61121003).

\section{References}

[1] Ian Dyer. Measurement of humidity. Anaesthesia \& Intensive Care Medicine, Volume: 13(2012): P. 121-123

[2] Z.M Rittersma. Recent achievements in miniaturised humidity sensors-a review of transduction techniques. Sensors and Actuators A, Volume: 96 (2002): P. 196-210

[3] Zhi Chen, Chi Lu. Humidity Sensors: A Review of Materials and Mechanisms. Sensor Letters, Volume: 3(2005): P. 274-295

[4] A. Robert Hillman. The EQCM: Electrogravimetry with a light touch. Journal of Solid State Electrochemistry, Volume 15(2011): P. 1647-1660

[5] Su-Yong Kwon, Yong-Gyoo Kim, and Inseok Yang. Study on the melting and freezing behaviour of high temperature binary eutectic fixed points using differential scanning calorimetry. Metrologia, Volume 47(2010): P. 248-252 